\section{Associação entre perda auditiva induzida pelo ruído e zumbidos}

\author{
Association between noise-induced \\ hearing loss and tinnitus
}

Adriano Dias 1,2
Ricardo Cordeiro 3
José Eduardo Corrente 4
Cláudia Giglio de Oliveira Gonçalves 5

\section{Introdução}

The objective of this study was to verify an association between noise-induced hearing loss (NIHL) and tinnitus in workers exposed to occupational noise. Workers with a history of work noise exposure who attended two audiology outpatient clinics were interviewed and evaluated. The statistical association between NIHL and tinnitus was evaluated using an adjusted linear regression model, with tinnitus as the dependent variable and NIHL, classified into six levels, as the independent variable, with age and length of noise exposure as covariates. Data were collected from 284 workers between April and October, 2003, in Bauru, São Paulo State, Brazil. Tinnitus was observed to increase with the progression of auditory damage, controlled by age and length of noise exposure. These results suggest that investment in hearing conservation programs is needed, especially for source noise emission control and for intervention in hearing loss progression that is caused by noise exposure, in order to maintain auditory health and reduce the associated symptoms.

Occupational Noise; Tinnitus; Hearing Losses
O ruído é o agente físico nocivo mais comum encontrado no ambiente de trabalho 1,2,3. Pela alta prevalência da exposição a intensidades deletérias à audição - em torno de $15 \%$ dos trabalhadores de países desenvolvidos, segundo a Organização Mundial da Saúde 4 - constitui-se em um importante agravo à saúde dos trabalhadores em todo o mundo.

A exposição ocupacional ao ruído intenso está associada a várias manifestações sistêmicas, tais como elevação do nível geral de vigilância, aceleração da freqüência cardíaca e respiratória, alteração da pressão arterial e da função intestinal, dilatação das pupilas, aumento do tônus muscular, aumento da produção de hormônios tireoidianos e estresse 5,6. Há também referências na literatura apontando associação possivelmente causal entre exposição ocupacional a ruído e acidentes do trabalho 7,8,9,10,11,12,13.

No ouvido, a exposição ocupacional ao ruído intenso lesa as células ciliares do órgão de Corti, causando perda progressiva e irreversível da audição, doença conhecida como perda auditiva induzida pelo ruído (PAIR). Ainda como manifestação auditiva, a exposição ocupacional ao ruído se associa ao zumbido, que também é chamado de acúfeno ou tínitus e pode ser definido como "uma ilusão auditiva, isto é, uma sensação sonora não relacionada com 
uma fonte externa de estimulação" 14 (p. 322). Sahley \& Nodar 15 (p. 323) o definem como " uma ocorrência na ausência de atividade vibratória ou mecânica correspondente nas orelhas média ou interna". Isso significa que o zumbido é uma percepção auditiva fantasma, que pode ser notada apenas pelo acometido na maior parte dos casos, o que dificulta sua mensuração padronizada. A fisiopatologia do zumbido é ainda controversa. Trata-se de um sintoma que produz extremo desconforto, de difícil tratamento, podendo, de acordo com sua gravidade, excluir do convívio social e até levar ao suicídio aqueles acometidos 16 .

Como contribuição ao entendimento da gênese do zumbido, o objetivo deste estudo foi verificar a associação entre PAIR e a queixa de zumbido em trabalhadores expostos ao ruído.

\section{Método}

Para este estudo transversal, a coleta das informações e os testes audiométricos foram realizados em dois ambulatórios de audiologia pertencentes à Universidade do Sagrado Coração, localizados na Cidade de Bauru, Estado de São Paulo, Brasil, com atendimento voltado a toda população. Foram avaliados e entrevistados 284 trabalhadores com histórico de exposição ao ruído ocupacional entre os meses de abril e outubro de 2003, encaminhados por médicos para avaliação audiológica.

Os indivíduos primeiramente assinaram o termo de consentimento, quando foram informados sobre a finalidade dos exames e como seriam utilizados os respectivos resultados. Após o consentimento, responderam à anamnese, da qual se extraíram antecedentes ocupacionais, riscos para deficiência auditiva e dados clínicos. Dentre as questões da anamnese, encontrava-se uma que se referia à presença ou ausência de zumbido.

Na avaliação clínica, procedeu-se à inspeção do meato acústico externo, que intencionou detectar presença de rolha de cerume, que, se constatada, contra-indicaria a realização dos exames, pois poderia simular algum grau de perda auditiva.

Após a inspeção do meato era realizada a audiometria tonal limiar, por via aérea nas freqüências de 250 e $500 \mathrm{~Hz}, 1,2,3,4,6$ e $8 \mathrm{kHz}$. Quando o limiar encontrado fosse igual ou maior que $25 \mathrm{~dB}$, também era realizada audiometria por via óssea nas freqüências de $500 \mathrm{~Hz}$, $1,2,3$ e $4 \mathrm{kHz}$. O procedimento para a testagem de ambas as vias foi idêntico, seguindo padrão ISO 17, utilizando-se de audiômetros calibra- dos de acordo com normas internacionais $18 \mathrm{e}$ respeitando tempo de repouso auditivo de, no mínimo, 14 horas. Os audiogramas eram, então, classificados de acordo com o critério proposto por Merluzzi et al. 19 .

Estudou-se a existência de associação entre PAIR e a ocorrência de zumbido por intermédio do ajuste de um modelo de regressão logística, tendo como variável dependente o zumbido $(\operatorname{sim}=1$, não $=0$ ) e como variável independente a PAIR, classificada como ausente (audiometria normal) ou perda auditiva de 1o, 2o, 3o, 4o e 5o graus. Foram excluídas as categorias Grau 6 e 7 de Merluzzi et al. 19, uma vez que elas não se referem à perda auditiva originada exclusivamente pelo ruído e também porque poucos trabalhadores neste estudo se enquadravam nessas categorias. No modelo, a classificação do audiograma foi codificada segundo cinco variáveis dummy em escala ordinal, sendo a categoria audiometria normal tomada como nível basal. A idade dos trabalhadores e o tempo de exposição ocupacional a ruído foram introduzidos no modelo como covariáveis.

Para o ajuste logístico, utilizou-se o programa SAS/STAT Software versão 0.2 (SAS Institute, Cary, Estados Unidos) no procedimento logistic. O Comitê de Ética em Pesquisa da Faculdade de Medicina de Botucatu, Universidade Estadual Paulista aprovou o protocolo deste estudo.

\section{Resultados}

Dentre os 284 trabalhadores entrevistados, 201 eram homens $(70,77 \%)$ e 83 eram mulheres $(29,23 \%)$. As idades variaram entre 20 e 72 anos (média de 42,51; mediana de 43 e desvio padrão de 12,63 anos) enquanto os tempos de exposição variaram entre 1 e 50 anos (média de 15,62; mediana de 15 e desvio padrão de 10,94 anos), constituindo-se numa casuística bastante heterogênea. Não houve recusas, e um único trabalhador foi encaminhado para retirada de rolha de cerume.

A Tabela 1 mostra a distribuição do total de indivíduos quanto à audição segundo a classificação de Merluzzi et al. 19. Para os ouvidos esquerdos, dos 284 trabalhadores, 106 (37\%) não apresentaram perda auditiva enquanto 178 (63\%) apresentaram algum grau de perda. Para os ouvidos direitos, 105 (37\%) não a apresentaram, mas 179 (63\%) apresentaram alguma alteração. Quanto ao zumbido, 149 trabalhadores (52\%) referiram não apresentá-lo, enquanto 135 (48\%) o percebiam em alguma intensidade. 
Distribuição dos indivíduos quanto à audição segundo Merluzzi et al. 19, para ambos os ouvidos, em estudo transversal. Bauru, São Paulo, Brasil, 2003.

\begin{tabular}{lrrrrrrrr}
\hline Merluzzi & \multicolumn{2}{c}{$\begin{array}{c}\text { Freqüência (n) } \\
\text { Esquerdo }\end{array}$} & \multicolumn{2}{c}{$\begin{array}{c}\text { \% } \\
\text { Direito }\end{array}$} & $\begin{array}{c}\text { Esquerdo } \\
\text { Eireito }\end{array}$ & $\begin{array}{c}\text { Freqüência acumulada } \\
\text { Esquerdo }\end{array}$ & $\begin{array}{c}\text { \% acumulado } \\
\text { Direito } \\
\text { Esquerdo } \\
\text { Direito }\end{array}$ \\
\hline Grau 0 & 106 & 105 & 37,32 & 36,97 & 106 & 105 & 37,32 & 36,97 \\
Grau 1 & 73 & 89 & 25,70 & 31,34 & 179 & 194 & 63,03 & 68,31 \\
Grau 2 & 41 & 32 & 14,44 & 11,27 & 220 & 226 & 77,46 & 79,58 \\
Grau 3 & 29 & 22 & 10,21 & 7,75 & 249 & 248 & 87,68 & 87,32 \\
Grau 4 & 11 & 8 & 3,87 & 2,82 & 260 & 256 & 91,55 & 90,14 \\
Grau 5 & 15 & 15 & 5,28 & 5,28 & 275 & 271 & 96,83 & 95,42 \\
Grau 6 & 4 & 9 & 1,41 & 3,17 & 279 & 280 & 98,24 & 98,59 \\
Grau 7 & 5 & 4 & 1,76 & 1,41 & 284 & 284 & 100,00 & 100,00 \\
\hline
\end{tabular}

A Tabela 2 mostra a distribuição dos trabalhadores quanto à ocupação.

Ao se ajustar o modelo de regressão logística, verificou-se associação significativa entre perda auditiva induzida pelo ruído e zumbido, controlada a idade e o tempo de exposição ao ruído. As estimativas dos parâmetros do modelo ajustado constam da Tabela 3, onde se nota que a categoria Merluzzi 1 (Grau 1) não se associa significativamente ao zumbido. Todas as demais categorias de Merluzzi analisadas associaram-se significativamente ao zumbido. É importante ressaltar que os ouvidos direitos, nas categorias de 2 a 5 , produziram sempre odds ratio maiores, chegando a mais que o dobro daquelas apresentadas pelos ouvidos esquerdos. Também na Tabela 3 são mostradas as odds ratio ajustadas para o modelo ordinal utilizado.

\section{Discussão}

A PAIR é considerada uma das doenças ocupacionais mais prevalentes em todo o mundo. Horg \& Raymond 20 encontraram PAIR em cerca de $60 \%$ dos 575 trabalhadores da construção civil avaliados nos Estados Unidos. Monley et al. 21 realizaram extensa coleta de informações audiológicas (89.500 sujeitos) da população australiana exposta ao ruído em níveis nocivos, encontrando prevalência de $57,7 \%$ de sujeitos com alterações auditivas sugestivas de indução pelo ruído. Andrade \& Schochat 22 avaliaram 7.043 trabalhadores expostos a ruídos intensos no $\mathrm{Mu}$ nicípio de São Paulo, encontrando prevalências entre 30 e 55\%, de acordo com o ramo de atividade. Também avaliando trabalhadores de diversos setores industriais da região metropolitana de Salvador (7.925 sujeitos de 44 indústrias),
Miranda et al. 23 encontraram prevalência geral de PAIR em torno de 36\%. Manubens 24 avaliou 32.007 trabalhadores de 150 indústrias de transformação brasileiras, encontrando a patologia em aproximadamente $23 \%$ dos indivíduos.

Além de exposições industriais, alguns estudos envolvendo trabalhadores expostos ao ruído em ambientes não industriais também foram encontrados. Cordeiro et al. 25 encontraram prevalência de PAIR em torno de $45 \%$ entre 292 motoristas e cobradores de veículos coletivos na Cidade de Campinas, enquanto Martins et al. 26, em estudo desenvolvido em Bauru, encontraram a patologia em $37 \%$ dos trabalhadores com o mesmo perfil profissional. Também com motoristas e cobradores em Campinas, Corrêa Filho et al. 27 encontraram prevalência de PAIR ao redor de $33 \%$.

Neste estudo, que envolveu trabalhadores expostos ao ruído sem distinção entre a exposição em ambiente industrial ou não industrial de acordo com o apresentado na Tabela 2 (isto é, a massa laboriosa como um grupo heterogêneo quanto à exposição), a prevalência encontrada foi de, aproximadamente, 63\%. Observese que os trabalhadores aqui avaliados são usuários de um serviço ambulatorial, não se tratando de uma amostra populacional probabilística. Assim sendo, a generalização da prevalência aqui encontrada para a população trabalhadora de Bauru como um todo é problemática. Entretanto, o objetivo deste estudo, conforme acima especificado, foi verificar a associação entre PAIR e zumbido. Se essa relação existir, ela se manifesta em trabalhadores expostos ocupacionalmente ao ruído, independentemente do desenho amostral 28.

Para o zumbido são escassos os dados epidemiológicos, ainda mais ao associá-lo a pato- 
Tabela 2

Distribuição dos indivíduos quanto à ocupação, em estudo transversal. Bauru, São Paulo, Brasil, 2003.

\begin{tabular}{|c|c|c|}
\hline Profissão & Freqüência & $\%$ \\
\hline Operador de máquinas & 30 & 10,56 \\
\hline Motorista & 28 & 9,86 \\
\hline Pedreiro & 21 & 7,39 \\
\hline Auxiliar de serviços gerais & 20 & 7,04 \\
\hline Auxiliar de cozinha & 18 & 6,34 \\
\hline Auxiliar odontológico & 16 & 5,63 \\
\hline Servente & 13 & 4,58 \\
\hline Marceneiro & 11 & 3,87 \\
\hline Odontólogo & 10 & 3,52 \\
\hline Auxiliar de lavanderia & 9 & 3,17 \\
\hline Mecânico & 9 & 3,17 \\
\hline Telefonista & 9 & 3,17 \\
\hline Costureiro & 7 & 2,46 \\
\hline Técnico em eletrônica & 7 & 2,46 \\
\hline Auxiliar de produção & 6 & 2,11 \\
\hline Carpinteiro & 6 & 2,11 \\
\hline Operador de cargas & 6 & 2,11 \\
\hline Cozinheiro & 5 & 1,76 \\
\hline Gráfico & 5 & 1,76 \\
\hline Pintor & 5 & 1,76 \\
\hline Atendente & 4 & 1,41 \\
\hline Cobrador & 4 & 1,41 \\
\hline Copeira & 3 & 1,06 \\
\hline Embalador & 3 & 1,06 \\
\hline Músico & 3 & 1,06 \\
\hline Soldador & 3 & 1,06 \\
\hline Vigilante & 3 & 1,06 \\
\hline Açougueiro & 2 & 0,70 \\
\hline Eletricista & 2 & 0,70 \\
\hline Funileiro & 2 & 0,70 \\
\hline Tecelã & 2 & 0,70 \\
\hline Almoxarife & 1 & 0,35 \\
\hline Armador & 1 & 0,35 \\
\hline Auxiliar de enfermagem & 1 & 0,35 \\
\hline Caldeireiro & 1 & 0,35 \\
\hline Ceramista & 1 & 0,35 \\
\hline Controlista & 1 & 0,35 \\
\hline Desinsetizador & 1 & 0,35 \\
\hline Jornalista & 1 & 0,35 \\
\hline Lavador & 1 & 0,35 \\
\hline Panificador & 1 & 0,35 \\
\hline Retificador & 1 & 0,35 \\
\hline Serralheiro & 1 & 0,35 \\
\hline Total & 284 & 100,00 \\
\hline
\end{tabular}

logias específicas. Sabe-se, contudo, que o zumbido grave é considerado o terceiro pior sintoma que pode acometer o ser humano, sendo superado apenas pelas dores e tonturas intensas e intratáveis 29.

A exposição ao ruído é responsável pela causa mais comum de zumbido ${ }^{29}$, referido por cerca de $25 \%$ dos indivíduos expostos 15 . Nesta casuística, a prevalência de zumbido encontrada ficou em torno de $48 \%$, muito superior àquela referida pela literatura. A subjetividade do sintoma é o fator que mais traz dificuldades e escassez de dados no estudo do zumbido, associada a alguns outros problemas, como: o fato de o zumbido ser sintoma e não doença; a inexistência de métodos de medição objetiva; a inexistência de modelos experimentais adequados e de que pode sofrer variações de acordo com o estado emocional ou físico do indivíduo 31.

Nesta pesquisa, a associação entre a PAIR e o zumbido foi identificada por intermédio de um modelo estatístico, levando em consideração a evolução do dano auditivo e a ocorrência do zumbido. Ao se constatarem os resultados, em especial a Tabela 3, conclui-se que não apenas existe associação entre PAIR e zumbido, mas também que há relação dose-resposta entre a perda auditiva e o zumbido.

No que se refere à idade, como pode ser observado na Tabela 3, o valor da odds ratio para ouvidos esquerdos foi 1,034 e para ouvidos direitos foi de 1,025 (embora com nível de significância entre $5 \%$ e $10 \%$ para este último). Isso significa que, para os trabalhadores estudados, a cada ano de vida, em média, aumenta a chance de ter zumbido em $3,4 \%$ e $2,5 \%$, nos ouvidos esquerdos e direitos, respectivamente.

Outro aspecto mostrado pela Tabela 3 e que chamou bastante a atenção foi o fato de as odds ratio encontradas para os ouvidos direitos nos graus de 2 a 5 serem sempre maiores que aquelas encontrados para os ouvidos esquerdos. Nenhum fator determinante que justificasse esta diferença foi encontrado dentre os dados coletados. Sugerem-se estudos de correlação anátomo-fisiológica para investigar possíveis diferenças que elucidem este achado, já que as exposições ao ruído investigadas nesta casuística não produziram pistas que conduzissem a essa explicação.

Os achados deste estudo justificam investimentos no controle da produção, emissão e transmissão do ruído nos postos de trabalho, utilização de proteção coletiva e individual contra este agente, avaliação periódica da audição e de sintomas associados, visando à preservação do bem-estar do trabalhador. 
Estimativa dos parâmetros do modelo de regressão logística ajustado e da associação entre o grau de perda auditiva segundo Merluzzi et al. 19 e o zumbido, para ambos os ouvidos, em estudo transversal. Bauru, São Paulo, Brasil, 2003*.

\begin{tabular}{|c|c|c|c|c|c|c|c|c|}
\hline \multirow[t]{2}{*}{ Parâmetro } & \multicolumn{2}{|c|}{ Estimativa } & \multicolumn{2}{|c|}{ Erro padrão } & \multicolumn{2}{|c|}{ Valor-p } & \multicolumn{2}{|c|}{ OR (IC95\%) } \\
\hline & Esquerdo & Direito & Esquerdo & Direito & Esquerdo & Direito & Esquerdo** & 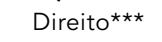 \\
\hline Intercepto & $-2,07$ & $-1,82$ & 0,49 & 0,51 & $<0,0001$ & 0,0004 & - & - \\
\hline Grau 1 & 0,44 & 0,35 & 0,32 & 0,3 & 0,1773 & 0,2565 & $\begin{array}{c}1,551 \\
(0,819-2,939)\end{array}$ & $\begin{array}{c}1,415 \\
(0,776-2,582)\end{array}$ \\
\hline Grau 2 & 0,78 & 1,43 & 0,40 & 0,46 & 0,0547 & 0,0021 & $\begin{array}{c}2,173 \\
(0,986-4,837)\end{array}$ & $\begin{array}{c}4,167 \\
(1,718-10,75)\end{array}$ \\
\hline Grau 3 & 1,41 & 1,6 & 0,48 & 0,58 & 0,0035 & 0,0057 & $\begin{array}{c}4,102 \\
(1,635-11,07)\end{array}$ & $\begin{array}{c}4,956 \\
(1,679-16,88)\end{array}$ \\
\hline Grau 4 & 1,73 & 2,47 & 0,84 & 1,1 & 0,0399 & 0,025 & $\begin{array}{c}5,635 \\
(1,25-39,9)\end{array}$ & $\begin{array}{c}11,789 \\
(1,921-227,4)\end{array}$ \\
\hline Grau 5 & 1,77 & 2,22 & 0,70 & 0,82 & 0,0111 & 0,0067 & $\begin{array}{c}5,893 \\
(1,654-27,89)\end{array}$ & $\begin{array}{c}9,202 \\
(2,193-63,45)\end{array}$ \\
\hline Idade & 0,03 & 0,03 & 0,01 & 0,01 & 0,0164 & 0,0897 & $\begin{array}{c}1,034 \\
(1,006-1,063)\end{array}$ & $\begin{array}{c}1,025 \\
(0,996-1,055)\end{array}$ \\
\hline $\begin{array}{l}\text { Tempo de } \\
\text { exposição }\end{array}$ & 0,002 & 0,01 & 0,01 & 0,01 & 0,8750 & 0,5972 & $\begin{array}{c}1,002 \\
(0,974-1,032)\end{array}$ & $\begin{array}{c}1,008 \\
(0,979-1,038)\end{array}$ \\
\hline
\end{tabular}

* Graus de liberdade $=1$;

** Odds ratio ajustadas para ouvidos direitos ( $\chi^{2}$ Razão de Verossimilhança $=51,6578$, 6 graus de liberdade, valor- $p<0,0001$ );

$\star \star \star$ Odds ratio ajustadas para ouvidos esquerdos ( $\chi^{2}$ Razão de Verossimilhança $=47,7815$,

6 graus de liberdade, valor-p $<0,0001)$.

\section{Resumo}

O estudo verificou a associação entre perda auditiva induzida por ruído (PAIR) e queixa de zumbido em trabalhadores expostos ao ruído ocupacional. Foram entrevistados e avaliados trabalhadores com histórico de exposição ao ruído ocupacional atendidos em dois ambulatórios de audiologia. Estudou-se a existência de associação entre PAIR e ocorrência de zumbido por intermédio do ajuste de modelo de regressão logística, tendo como variável dependente o zumbido e como variável independente a PAIR, classificada em seis graus, controlada pelas co-variáveis idade e tempo de exposição ao ruído. Os dados foram coletados entre abril e outubro de 2003, na Cidade de Bauru, Estado de São Paulo, Brasil, contemplando 284 trabalhadores. Estimou-se que a prevalência de zumbido aumenta de acordo com a evolução do dano auditivo, controlado para a idade e tempo de exposição ao ruído. Os achados justificam o investimento em programas de conservação auditiva particularmente voltados para o controle da emissão de ruídos na fonte e para a intervenção na evolução das perdas auditivas geradas pela exposição ao ruído visando à manutenção da saúde auditiva e à diminuição dos sintomas associados.

\section{Colaboradores}

A. Dias participou do planejamento global do estudo, coleta de dados, análise global dos dados e redação do texto. R. Cordeiro participou do planejamento global do estudo, análise global dos dados e redação do texto. J. E. Corrente participou da análise estatística dos dados e redação do texto. C. G. O. Gonçalves participou da análise dos dados audiométricos. 


\section{Referências}

1. Alberti PW. Deficiência auditiva induzida pelo ruído. In: Lopes Filho O, Campos CAH, organizadores. Tratado de otorrinolaringologia. São Paulo: Roca; 1994. p. 934-49.

2. Seligman J. Sintomas e sinais na PAIR. In: Nudelmann AA, Costa EA, Seligman J, Ibañez RN, organizadores. PAIR: perda auditiva induzida pelo ruído. Porto Alegre: Baggagem; 1997. p. 143-51.

3. Miranda CR. Introdução à saúde no trabalho. São Paulo: Atheneu; 1998.

4. World Health Organization. Occupational and community noise. Geneva: World Health Organization; 2001. (Fact Sheet 258).

5. Cohen A. Extra-auditory effects of occupational noise - Part II: effects on work performance. Natl Saf News 1973; 109:68-76.

6. Costa EA, Kitamura S. Órgãos dos sentidos: audição. In: Mendes R, organizador. Patologia do trabalho. Rio de Janeiro: Atheneu; 1995. p. 365-87.

7. Barreto SM, Swerdlow AJ, Smith PG, Higgins CD. A nested case-control study of fatal work related injuries among Brazilian steel workers. Occup Environ Med 1997; 54:599-604.

8. Berger EH, Royster LH, Royster JD, Driscoll DP, Layne M. The noise manual. $5^{\text {th }}$ Ed. Akron: American Industrial Hygiene Association; 2000.

9. Cohen A. Industrial noise and medical absence and accident record data on exposed workers. In: Proceedings of the International Congress on Noise as a Public Health Problem. Washington DC: US Environmental Protection Agency; 1976. p. 441-53.

10. Cordeiro R, Clemente APG, Diniz SC, Dias A. Exposição ao ruído ocupacional como fator de risco para acidentes do trabalho. Rev Saúde Pública 2005; 39:461-6.

11. Kjeliberg A. Subjective, behavioral and psychophysiological effects of noise. Scand J Work Environ Health 1990; 16:29-38.

12. Melamed S, Luz J, Green MS. Noise exposure, noise annoyance and their relation to psychological distress, accident and sickness absence among blue-collar workers - the Cordis Study. Isr J Med Sci 1992; 28:629-35.

13. Moll-van Charante A, Mulder PGH. Perceptual acuity and the risk of industrial accidents. Am J Epidemiol 1990; 131:652-63.

14. Bento RF, Miniti A, Marone SAM. Doenças do ouvido interno. In: Bento RF, Miniti A, Marone SAM, organizadores. Tratado de otologia. São Paulo: Edusp; 1998. p. 322-31.

15. Sahley TL, Nodar RH. Tinnitus: present and future. Curr Opin Otolaryngol Head Neck Surg 2001; 9:323-8.

16. Hallam RS, Rachman S, Hinchcliff R. Psychological aspects of tinnitus. In: Rachman R, editor. Contributions to medical psychology. Oxford: Pergamon Press; 1984. p. 31-4.

17. International Organization for Standardization. Basic pure tone air and bone conduction threshold audiometry: ISO 8253-1. Geneva: International Organization for Standardization; 1989.
18. American National Standard Institute. Specification for audiometers: S3.6-1996. New York: Acoustical Society of America; 1996.

19. Merluzzi F, Cornacchia L, Parigi G, Terrana T. Metodologia di esecuzione del controllo dell'udito dei lavoratori esposti a rumore. Nuovo Archivio Italiano di Otologia, Rinologia e Laringologia 1979; 7:695-714.

20. Horg OS, Raymond DM. How serious is hearing loss among US construction workers? In: Anais do XXVII International Congress on Occupational Health [CD-ROM]. Foz do Iguaçu: International Commission on Occupational Health; 2003.

21. Monley P, West A, Guzeleva D, Dinh DA, Tzvetkova J. Hearing impairment in the western Australian noise exposed population. Aust J Audiol 1996; 18:59-71.

22. Andrade CRF, Schochat E. Perfil audiométrico de trabalhadores de indústrias ruidosas. In: Anais do I Encontro Nacional de Fonoaudiologia Social e Preventiva. São Paulo: Conselho Regional de Fonoaudiologia 2a Região; 1988. p. 71-81.

23. Miranda CR, Dias CR, Pena PGL, Nobre LCC, Aquino, R. Perda auditiva induzida pelo ruído em trabalhadores da região metropolitana de Salvador, Bahia. Inf Epidemiol SUS 1998; 7:87-94.

24. Manubens RS. Avaliação médico-ocupacional das perdas auditivas em trabalhadores expostos a ruído em indústrias brasileiras. In: Anais do I Simpósio Brasileiros de Surdez Ocupacional. São Paulo: Sociedade Brasileira de Otorrinolaringologia; 1994. p. 28-9.

25. Cordeiro R, Lima-Filho EC, Nascimento LCR. Associação da perda auditiva induzida pelo ruído com o tempo acumulado de trabalho entre motoristas e cobradores. Cad Saúde Pública 1994; 10:210-21.

26. Martins AL, Alvarenga KF, Bevilacqua MC, Costa Filho OA. Perda auditiva em motoristas e cobradores de ônibus. Rev Bras Otorrinolaringol 2001; 67:467-73.

27. Corrêa Filho HR, Costa LS, Hoehne EL, Pérez MAG, Nascimento LCR, Moura EC. Perda auditiva induzida por ruído e hipertensão em condutores de ônibus. Rev Saúde Pública 2002; 36:693-701.

28. Miettinen OS. Theoretical epidemiology: principles of occurrence research in medicine. New York: John Wiley \& Sons; 1985.

29. Sanchez TG, Bento RF, Miniti A, Câmara J. Zumbido: características e epidemiologia. Experiência do Hospital das Clínicas da Faculdade de Medicina da Universidade de São Paulo. Rev Bras Otorrinolaringologia 1997; 63:229-35.

30. Andersson G, Vretblad P, Larsen HC, Lyttkens L. Longitudinal follow-up of tinnitus complaints. Arch Otolaryngol Head Neck Surg 2001; 127:175-9.

31. Jastreboff PJ, Hazell JWP. A neurophysiological approach to tinnitus: clinical implications. Br J Audiol 1993; 27:7-17.

Recebido em 17/Nov/2004

Versão final reapresentada em 21/Mai/2005 Aprovado em 24/Jun/2005 